\title{
Construção da Taxonomia Brasileira para Descritores da Personalidade
}

\author{
Raquel Soura Lobo Guzzo ${ }^{12}$ \\ Pontificia Universidade Católica de Campinas \\ Cristina Coutinho Marques de Pinho \\ Pontificia Universidade Católica de Campinas \\ Carolina Freire de Carvalho de Carvalho \\ Pontificica Universidade Católica de Campinas
}

\begin{abstract}
Resumo
Esta é uma pesquisa que utiliza a abordagem psicoléxica para servir de apoio aos estudos sobre avaliação da personalidade. Trata-se de um projeto, seguindo a metodologia alemã como parte de um programa transcultural. A importância deste estudo refere-se, principalmente, ao estabelecimento de uma base científica para a construção ou adaptação de instrumentos para avaliação psicológica. O projeto inclui quatro fases: 1) identificação e extração de adjetivos existentes no léxico da língua portuguesa; 2) exclusão de adjetivos de acordo com critérios estabelecidos pela proposta alemã, em uma análise de dois juízes; 3) classificação dos adjetivos quanto à freqüência de uso, à clareza de significado e à utilidade como descritor da personalidade, em uma análise de 6 juízes; 4- classificação dos adjetivos restantes por dez juízes diante de categorias definidas pela proposta alemã. O presente trabalho pretende relatar os resultados das fases um e dois e fazer uma comparação com diferentes culturas que também desenvolveram estudos semelhantes (italiana, tcheca e alemã). Foram extraídos do Dicionário Aurélio da Língua Portuguesa, 35.834 adjetivos de um total de 120 mil verbetes, representando 29,86\% do total de vocábulos. Após a primeira análise, permaneceram 16,03\% dos adjetivos. Quanto à comparação entre os diferentes resultados dos países, o Brasil apresenta maior número de adjetivos do léxico, porém, os resultados da Alemanha incluem mais adjetivos descritores da personalidade.

Palavras-chave: Taxonomia; personalidade; avaliação psicológica.
\end{abstract}

Construction of Brazilian Taxonomy for Personality's Descriptors

Abstract

This is a research using the psycolexical approach to support studies about assessment of personality. The importance of this project is to create a scientific basis for the construction of instruments in personality assessment. It follows Germany's methodology and has four steps: 1) to identify and extract from the dictionary all adjectives presented;2) selection of those adjectives which are descriptors of personality, 3) classification, with six judges, of those adjectives considering frequency, clear of meaning and utility for personality description, 4) classification in specific categories using 10 judges. The present work intends to relate steps one and two and make a comparison with different results from cultures, including Germam, Tchecan and Italian. There are 35.834 adjectives considering a total of 120,000 words, representing $29.86 \%$. From this number, $16.03 \%$ are considered personality descriptors, by two judges. Brazil presents the largest number of adjectives in lexicon, but Germany presents more adjectives descriptors of personality. Keywords: Taxonomy; personality; psychological assessment.

Os estudos referentes à avaliação psicológica no Brasil apresentam dificuldades básicas em seu desenvolvimento. Um dos principais problemas apresentados pelos autores diz respeito à qualidade e quantidade de instrumentos de avaliação disponíveis para a realidade brasileira. (Andriola,

1 Endereço para correspondência: R. Santa Mônica 136, 13094-531, Campinas, SP. Fone (19) 3251.4206, Fax (19) 3255.1970. E-mail rguzzo@mpc.com.br. 2 Projeto financiado pelo CNPq.

3 Versão anterior deste trabalho foi apresentado no IV Congresso Nacional de Psicologia Escolar e Educacional, em abril de 1998.
1996; Hutz \& Bandeira, 1993; Pasquali, 1992). O processo de construção de instrumentos de avaliação é complexo, trabalhoso e, muitas vezes, caro. Se, de uma maneira geral, a Psicologia brasileira não dispõe de suporte tecnológico para avaliação, de uma forma específica, na área da personalidade se acentuam estas dificuldades.

Personalidade é um constructo da Psicologia que se destaca pela dificuldade de ser definido e estudado, apesar de ser um dos mais antigos aspectos pesquisados do ser humano. Sendo assim, a avaliação da personalidade deve ser criteriosa para não impedir o conhecimento do 
funcionamento e comportamento humano, a partir das diferenças individuais. $\mathrm{O}$ instrumento, portanto, deve ser capaz de avaliar e descrever tais características.

Cloninger (1996) constatou que as perspectivas contemporâneas da personalidade têm se baseado no estudo dos traços e têm criado um modelo amplo de organização para estruturá-los. Angleitner e Ostendorf (1994) apontaram que o desenvolvimento de taxonomias de traços de personalidade permite a descrição das características individuais, de forma cada vez mais segura e importante. Os objetivos principais da busca por descritores da personalidade, através da taxonomia, são encontrar palavras adequadas para cada diferença individual e agrupar um conjunto de termos que representem características individuais e de grupos.

A partir deste pressuposto, a abordagem léxica, ou psicoléxica, foi desenvolvida com a finalidade de abranger os termos que descrevessem a personalidade de um indivíduo, identificando, agrupando e classificando as palavras que são mais representativas na linguagem diária (Angleitner, Ostendorf \& John, 1990; De Raad, 1995; Fujita, s/d; John, Angleitner \& Ostendorf, 1988).

Os estudos referidos abaixo sustentam que a abordagem léxica considera que as diferenças individuais mais expressivas e socialmente relevantes para a vida cotidiana estão incorporadas na linguagem. A linguagem constitui-se de palavras e expressões que representam características situações constantes e significativas da vida dos indivíduos. Quanto mais claramente tais diferenças se constituem, mais tendem a ser representadas lingüisticamente por uma única palavra. Desta forma, a abordagem léxica converge para o domínio do traço, uma vez que as pessoas, na sua comunicação diária, tratam das diferenças individuais por meio da linguagem (Fujita, s/d; Goldberg, 1982; John \& cols.,1988; John, Goldberg \& Angleitner, 1984). Est abordagem apresenta, na visão de Fujita (s/d), a vantagem de ser ateórica, oferecendo uma estrutura comum para diferentes abordagens. O desafio de se encontrar uma expressão adequada para cada diferença entre as pessoas e constructos e, ao mesmo tempo, agrupar um conjunto de palavras e expressões que representem características individuais, tem dirigido a atenção de estudiosos para pesquisas de descritores da personalidade (Angleitner \& cols., 1990; De Raad, 1995).

As diferenças individuais estão representadas no léxico na forma de verbos, adjetivos, substantivos, substantivos adjetivados, etc., uma vez que neste está representado o conjunto de todas as palavras que as pessoas podem usar para a comunicação. As características individuais relevantes para o estudo de descritores da personalidade dependem, basicamente, de dois fatores. O primeiro deles é a freqüência, pois quanto maior a freqüência com que as pessoas falam sobre um traço, mais importante ele é considerado e utilizado naquela população. $O$ segundo fator é o tempo em que certas unidades permanecem em uso (Angleitner e cols., 1990). Devido à quantidade de verbetes existentes no dicionário, nem sempre é possível identificar termos descritores da personalidade sem considerar a clareza de significado da palavra possivelmente um terceiro fator relevante.

A identificação de descritores da personalidade na linguagem tem levado a estudos que buscam listar quais são as dimensões que as pessoas usam para descreverem a si mesmas e os outros, através de uma taxonomia própria da personalidade. Os procedimentos para a construção de uma taxonomia de descritores da personalidade passaram a ser uma importante base de dados para a construção de instrumentos de avaliação da personalidade e temperamento (Angleitner \& Ostendorf, 1994; Eysenk, 1994; Ostendorf, 1994; Schmitz, 1994; Szirmák, 1994). Fujita (s/d) considera que "uma taxonomia mal feita ainda é melhor que nenhuma" (p. 1), destacando a importância de uma organização e estabelecimento de uma base científica na avaliação psicológica, principalmente, na avaliação da personalidade.

Diferentes países têm participado de um projeto transcultural sobre o desenvolvimento de uma taxonomia de descritores da personalidade, tais como Itália (Forzi, Arcuri, Fontana, Di Blas \& Tortul, 1990), Alemanha (Angleitner e cols., 1990), Tchecoslováquia ${ }^{4}$ (Hrebíckovà, Ostendorf \& Angleitner, 1994), Hungria (Szirmák, 1994), E.U.A. (Norman, 1967 citado por John, Goldberg \& Angleitner, 1984), Holanda (Broken, 1978; citado por Hofstee, 1990) e, a partir deste trabalho, Brasil (Guzzo, Riello, Pinho, Carvalho \& Koelle, 1998). Os países envolvidos neste projeto transcultural estão buscando um padrão de categorias, atualmente caracterizado de três a seis agrupamentos de características individuais presentes nas diferentes culturas, pois acredita-se que independente da língua de um povo, determinados fatores estão presentes e podem ser agrupados em categorias comuns (Ostendorf \& Angleitner, 1992).

Esta é a primeira pesquisa no Brasil a utilizar a abordagem psicoléxica para servir de apoio aos estudos sobre avaliação da personalidade. É um estudo baseado na proposta alemã do desenvolvimento da criação da taxonomia (Angleitner, e cols., 1990) que pretende contribuir para o projeto transcultural, favorecer estudos teóricos sobre personalidade, aprimorar técnicas de avaliação psicológica e identificar os termos descritores da personalidade na realidade brasileira.

${ }^{4}$ A referência utilizada é datada anteriormente a divisão das duas Repúblicas (Tcheca e Eslováquia). 
O desenvolvimento da taxonomia deve ser realizado em quatro etapas, como sugere a versão alemã: a) extração dos termos descritores da personalidade; b) primeira análise de juízes na verificação dos termos; c) avaliação por seis juízes quanto à clareza de significado, freqüência de uso e utilidade como descritor; e, d) categorização dos termos, por dez juízes. O estudo brasileiro encontra-se atualmente no início da terceira fase, tendo sido desenvolvidas, no Laboratório de Avaliações e Medidas Psicológicas (LAMP da PUC Campinas), as duas primeiras etapas. O presente trabalho apresentará o desenvolvimento das duas etapas iniciais para a construção de uma taxonomia de descritores da personalidade na realidade brasileira.

A primeira fase consistiu da extração de todos os adjetivos encontrados no dicionário da língua portuguesa (Barroso, 1996). Apesar de alguns estudos (Hofstee, 1990; Wiggins \& Pincus, 1992) reprovarem a avaliação de termos descritores da personalidade só através de adjetivos, De
Raad (1995), Angleitner e colaboradores (1990) indicam que estes termos são suficientes para se fazer uma primeira classificação.

O material utilizado foi o Dicionário Aurélio Eletrônico, versão $2.0 \mathrm{em}$ cd-rom, (Barroso, 1996). O procedimento foi selecionar todos os verbetes nos quais apareciam a sigla $\mathrm{ADJ}$, copiá-los e "colá-los" em um arquivo do processador de texto.

Segundo o Novo Dicionário Aurélio (Ferreira, 1986), existem cento e vinte mil verbetes. A primeira fase deste estudo constatou que, deste total, 35.834 vocábulos são adjetivos, representando $29,86 \%$ do total de verbetes existentes no léxico. Pode-se considerar expressiva esta quantidade de adjetivos, uma vez que quase $70 \%$ correspondem a todas as outras categorias (verbos, advérbios, substantivos, etc.). Do total de adjetivos, aqueles considerados descritores da personalidade são 5.744 $(4,79 \%)$.

Tabela 1 . Total de Adjetivos Extraídos do Dicionário por Letra

\begin{tabular}{|c|c|c|c|c|}
\hline Letras & $\begin{array}{l}\text { Total de } \\
\text { adjetivos }\end{array}$ & $\%$ & $\begin{array}{l}\text { Total de adjetivos } \\
\text { descritores }\end{array}$ & $\%$ \\
\hline $\bar{A}$ & 4725 & 13,19 & 826 & 16,97 \\
\hline B & 1452 & 4,05 & 236 & 16,25 \\
\hline $\mathrm{C}$ & 3645 & 10,17 & 529 & 12,89 \\
\hline $\mathrm{D}$ & 1985 & 5,54 & 521 & 26,34 \\
\hline $\mathrm{E}$ & 2618 & 7,31 & 286 & 10,84 \\
\hline $\mathrm{F}$ & 1403 & 3,92 & 296 & 21,09 \\
\hline G & 984 & 2,75 & 111 & 11,38 \\
\hline $\mathrm{H}$ & 895 & 2,50 & 123 & 13,63 \\
\hline I & 2307 & 6,44 & 689 & 29,99 \\
\hline $\mathrm{J}$ & 280 & 0,78 & 40 & 14,28 \\
\hline $\mathrm{K}$ & 11 & 0,03 & 0 & - \\
\hline $\mathrm{L}$ & 1066 & 2,97 & 123 & 15,76 \\
\hline $\mathrm{M}$ & 2188 & 6,11 & 287 & 13,16 \\
\hline $\mathrm{N}$ & 708 & 1,98 & 122 & 17,23 \\
\hline $\mathrm{O}$ & 894 & 2,49 & 116 & 13,08 \\
\hline $\mathrm{P}$ & 3414 & 9,53 & 410 & 12,62 \\
\hline Q & 247 & 0,69 & 51 & 20,64 \\
\hline $\mathrm{R}$ & 1649 & 4,60 & 279 & 16,01 \\
\hline S & 2136 & 5,96 & 321 & 15,03 \\
\hline $\mathrm{T}$ & 1731 & 4,83 & 197 & 28,77 \\
\hline $\mathrm{U}$ & 398 & 1,11 & 22 & 5,52 \\
\hline $\mathrm{V}$ & 796 & 2,22 & 122 & 15,32 \\
\hline $\mathrm{X}$ & 121 & 0,34 & 18 & 14,87 \\
\hline $\mathrm{Y}$ & 0 & 0,00 & 0 & - \\
\hline W & 10 & 0,03 & 0 & - \\
\hline $\mathrm{Z}$ & 171 & 0,48 & 19 & 11,11 \\
\hline TOTAL & 35834 & $100 \%$ & 5744 & 16,03 \\
\hline
\end{tabular}

Psicologia: Reflexão e Crítica, 2002, 15(1), pp. 71-75 
A Tabela 1 demonstra, ainda, a distribuição da quantidade de adjetivos por letra do alfabeto. Existe uma variação desta quantidade por letra, sendo a máxima 4.725 (letra A) e a mínima 10 (letra W).

Sabe-se que as letras $\mathrm{K}, \mathrm{W}$ e Y não fazem parte do alfabeto do Brasil e que são utilizadas apenas para palavras estrangeiras. Contudo, destas, a única letra que não contém adjetivos é a Y. As letras $\mathrm{K}$ e W apresentam um baixo número de adjetivos, 11 e 10 respectivamente, apesar de nenhum deles serem descritores da personalidade (ver Tabela 1). As letras X e Z contêm números de adjetivos inesperados (121 e 171, respectivamente) para a língua portuguesa, apesar de apresentarem baixa freqüência relativa $(0,34 \%$ e $0,48 \%)$.

Pode-se observar que as letras mais representativas em termos de quantidade de adjetivos são, em ordem de grandeza, A $(13,19 \%), C(10,17 \%)$ e P $(9,53 \%)$. Por estrutura da Língua Portuguesa, as adjetivações com conotação negativa são mais representativamente freqüentes nas letras A, D e I (por exemplo, "anormal", "desorganizado" “inadequado"). A Tabela 1 apresenta os resultados da primeira fase na construção da taxonomia brasileira.

$\mathrm{Na}$ segunda fase foi feita uma seleção dos adjetivos retirados do dicionário, com a análise de dois juíze selecionados dentre os bolsistas de Iniciação Científica do Laboratório de Avaliação e Medidas Psicológicas da PUC Campinas, e segundo critérios de exclusão pré-estabelecidos pela proposta alemã. Os critérios de exclusão referiram-se aos termos que: aplicavam-se a: a) todas as pessoas (ex. humano); b) representavam origem geográfica ou nacionalidade (ex.: baiano, brasileiro); c) indicavam profissão ou atividade (ex.: catequista); d) ressaltavam uma parte da pessoa (ex.: barrigudo); e) eram considerados metáforas (ex quérulo); f) apresentavam aspectos técnicos e científicos (ex.: cacotímico); g) expressavam idéias políticas, religiosas ou filosóficas (ex.: capitalista, ateu, marxista); h) eram relativos à natureza (ex.: cariofiláceo); i) eram considerados chulos; i) retratavam a constituição física do indivíduo (ex.: manco); 1) eram relativos a animais (ex.: cansão).

Cada juiz trabalhou independentemente, na decisão de excluir os adjetivos pelos diferentes critérios. A exclusão de um adjetivo foi determinada quando os dois juízes concordaram com a retirada. Quando havia dúvida em relação a permanência ou não do adjetivo, optou-se por mantê-lo (norma alemã).

A Tabela 1 indica o total de adjetivos considerados descritores da personalidade por letra, totalizando 5.744 termos, representando $16,03 \%$ do total de adjetivos e $4,79 \%$ da quantidade total de palavras existentes no léxico. A porcentagem descrita na Tabela 1 está relacionada ao número de adjetivos da letra, destacando a letra I como aquela que apresenta mais adjetivos descritores da personalidade no conjunto de adjetivos $(29,87 \%)$.

A partir destes resultados, pôde-se fazer uma comparação com os resultados de alguns países envolvidos no projeto transcultural de taxonomia de descritores da personalidade com procedimentos semelhantes, apresentada na Tabela 2 (Angleitner e cols., 1990; Forzi e cols., 1990; Guzzo e cols., 1998; Hrebíckovà e cols., 1994).

Observa-se uma variação no total de verbetes presentes nos léxicos alemão, italiano, brasileiro e tcheco. Quanto ao total de adjetivos presentes na Língua Portuguesa, o Brasil apresenta o maior número (35.834), seguido pela língua italiana (21.800), tcheca (13.606) e alemã (11.600). Porém, na conclusão da segunda fase, os resultados da Alemanha incluem, proporcionalmente, mais adjetivos descritores da personalidade (42\%), principais para a construção da taxonomia. (Tabela 2).

Embora a proporção de descritores da personalidade com relação à quantidade de adjetivos seja maior na taxonomia alemã, com relação ao total de verbetes essa proporção é a mesma para a Alemanha e o Brasil. Ou seja, $5 \%$ das palavras existentes nos dicionários alemão e brasileiro são adjetivos descritores da personalidade.

Não se pode chegar, neste momento, a conclusões definitivas, uma vez que o projeto ainda não está finalizado. Deve-se ressaltar, no entanto, que este é o início da construção de uma taxonomia de descritores da personalidade cujas fases seguintes devem aprimorar ainda mais o processo de identificar aqueles vocábulos que servirão para descrever, de forma mais precisa, diferenças individuais. Importante notar que a Língua Portuguesa apresenta uma variabilidade

Tabela 2. Comparação do Total de Adjetivos em Diferentes Países

\begin{tabular}{lccccc}
\hline Países & $\begin{array}{c}\text { Total de } \\
\text { verbetes }\end{array}$ & $\begin{array}{c}\text { Total de } \\
\text { adjetivos }\end{array}$ & $\%$ & $\begin{array}{c}\text { Total de adjetivos descritores da } \\
\text { personalidade }\end{array}$ & $\%$ \\
Alemanha & 96.666 & 11.600 & 12 & 4.437 & 42 \\
Itália & 127.000 & 21.800 & 17 & 5.145 & 14 \\
Tchecoslováquia & 64.800 & 13.606 & 21 & & 30 \\
Brasil & 120.000 & 35.834 & 30 & & 16 \\
\hline
\end{tabular}


incontestável de adjetivos passíveis desta descrição, e que dificuldades na avaliação psicológica aparecem, precisamente, pelo fato de não se poder assegurar a análise destas características pelas dificuldades no uso da linguagem. Diante disto, a importância da taxonomia se coloca com ferramenta fundamental para o desenvolvimento de pesquisa na área de avaliação psicológica, sobretudo da personalidade.

\section{Referências}

Andriola,W. B. (1996). Avaliação psicológica no Brasil: Consideraçoes a respeito da formação dos psicólogos e dos instrumentos utilizados. Psique, 6(8), 98-108.

Angleitner, A. \& Ostendorf, F. (1994). Von aaglatt bis Zynisch: Merkmale persönlichkeitsbescreibender begriffe. Em W. Hager \& M. Hasselhor (Orgs.), Handbucb deutschsprachiger wort normen (pp 38-62). Göttingen: Hogrefe.

Angleitner, A., Ostendorf, F. \& John, O. P. (1990). Towards a taxonomy of personality in german: A psycholexical study. European Journal of Personality, 4, 89-118.

Barroso, M. E. G. (1996). Dicionário eletrônico Aurélio versão 2.0 em CD-Rom.

Cloninger, S.C. (1996). Personality: Description, dynamics and development. New York: W. H. Freeman \& Company.

De Raad, B. (1995). The psycholexical approach to the structure of interRaad, B. (1995). The psycholexical approach to the struct
personal traits. European Journal of Personality, 9, 89-102.

Eysenck, H. J. (1994). The importance of theory in the taxonomy of personality. Personality Psychology in Europe, 5, 243-252.

Ferreira, A. B. H. (1986). Novo Dicionário Aurélio da Lingua Portuguesa. Rio de Janeiro: Nova Fronteira.

Forzi, M., Arcuri, L., Fontana, R. M., Di Blas, L. \& Tortul, M. (1990, Julho). Towards a taxonomy of italian personality-descriptive terms, trabalho apresentado at the $5^{\text {th }}$ EAPP Conference on Personality. Roma, Itália.

Fujita, F. (s/d). The big five taxonomy, based on a qualifying exam answer. Retirado de http: \\www.iusb.edu/ ffujita/Documentos/ big 5. html, em 10/10/2000.

Goldberg, L. R. (1982). From ace to zombie: Some exploration in the language of personality. Em C. D. Spielberg \& J. N. Butcher (Orgs.), Advances in personality assesment (pp. 73-89). Hilsdale: Erlbaun.

Guzzo, R. S. L. Carvalho C F. C Messias, T. S. C Pinho C. C. M., Gums, E. F., Pereira, P.C., Riello, I. C., Scoz, M. C. P. \& Serrano, M. R. (1998). Construção da taxonomia brasileira para descritores da personalidade: Um estudo piloto [Resumos]. Em Associação Brasileira de Psicologia Escolar e Educacional (Org.), Anais do IV Congresso Nacional de Psicologia Escolar (ABRAPEE), (p.111). João Pessoa, PB: ABRAPEE.
Guzzo, R. S. I. Riello, I. C. Pinho C C. M Carvalho, C. F. C \& Koelle, G A. (1998). Taxonomia para descritores da personalidade: Comparando diferentes culturas [Resumos]. Em IV Conferenncia Internacional: Avaliagáa Psicologica. Formas e Contextos (p. 141). Salamanca, Espanha.

Hofstee, W. K. B. (1990). The use of everyday personality language for scientific purpose. European Journal of Personality, 4, 77-88

Hrebícková, M., Ostendorf, F. \& Angleitner, A. (1994). Sawische und germanishe persönlinchkeitssprache: Vergleich der egerbmisse eine tschechischen und einer deutschen taxonomie. Kongress der Deutscen Gesellschanft für Psychologie (p.1-11) Göttingen.

Hutz, C. S. \& Bandeira, D. R. (1993). Tendências contemporâneas no uso de testes: Uma análise da literatura brasileira e internacional. Psicologia: Reflexão e Crítica, 6, 85-101.

John, O. P., Angleitner, A. \& Ostendorf, F. (1988). The lexical approach to personality: A historical review of trait taxonomic research. European Journal of Personality, 2, 171-203.

John, O. P., Goldberg, L. R \& Angleitner, A. (1984). Better than the alphabet Taxonomies of personality-descriptive terms in English, Deutsch and German. Em H. C. J. Bonarius, G. L. M. van Heck \& N. G. Smid (Orgs.), Personality psychology in Europe: Theorical and empirical development (pp. 41-53). Lisse, N.L.: Swets and Zeitlinger.

Ostendorf, F. (1994). Zur taxonomie deutscher dipositionbegriffe. Em

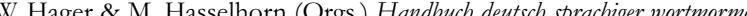
(118-138). Göttingen: Hogrefe.

Ostendorf, F. \& Angleitner, A. (1992). On the generality and comprehensiveness of five-factor model of personality: Evidence for five robust factors in questionannaire data. Em G.V. Caprara \& G.L. van Heck (Org.), Modern Personality Psychology. Critical Reviews and New Direction. (97-110). Harvester-Wheatscheaf.

Pasquali, L. (1992). Avaliação psicológica: Questões e controvérsias [Resumos]. Em Associação Brasileira de Psicologia Escolar e Educacional (Org.), Anais do I Congresso Nacional de Psicologia Escolar (p. 61-63). Campinas, SP: ABRAPEE.

Schmitz, P. G. (1994). Dimensions of personality in free description. Personality Psychology in Europe, 5, 319-322.

Szirmák Z (1994). Learning the alphabet: Constructing the list of hungarian personality descriptive terms. Personality Psychology in Europe, 5, 278-

Wiggins, J. S. \& Pincus, A. L. (1992). Personality: Structure and assessment. Annual Review of Psychology, 43, 473-504.

Sobre as autoras

Raquel Souza Lobo Guzzo é Psicóloga, Professora Titular do Instituto de Psicologia e Fonoaudiologia da Pontifícia Universidade Católica de Campinas, Doutora em Psicologia Escolar pelo Instituto de Psicologia da USP, São Paulo.

Cristina Coutinho Marques de Pinho é Psicóloga, Mestranda em Psicologia Escolar pelo Instituto de Psicologia e Fonoaudiologia da Pontifícia Universidade Católica de Campinas, bolsista CAPES/

Carolina Freire de Carvalho de Carvalho é Estudante de Graduação em Psicologia no Instituto de Psicologia e Fonoaudiologia da Pontifícia Universidade Católica de Campinas, bolsista PIBIC/ CNPq. 